\title{
Syntax, Semantics, and Pragmatics of Contexts
}

\author{
John F. Sowa \\ Philosophy and Computers and Cognitive Science \\ State University of New York at Binghamton
}

\begin{abstract}
The notion of context is indispensable in discussions of meaning, but the word context has often been used in conflicting senses. In logic, the first representation of context as a formal object was by the philosopher C. S. Peirce; but for nearly eighty years, his treatment was unknown outside a small group of Peirce aficionados. In the early 1980s, three new theories included related notions of context: Kamp's discourse representation theory; Barwise and Perry's situation semantics; and Sowa's conceptual graphs, which explicitly introduced Peirce's approach to the AI community. More recently, John McCarthy and his students have begun to use a closely related notion of context as a basis for organizing and partitioning knowledge bases. Each of the theories has distinctive, but complementary ideas that can enrich the others, but the relationships between them are far from clear. This paper analyzes the semantic foundations of these theories and shows how McCarthy's ist $(c, p)$ predicate can be interpreted in terms of the semantic notions underlying the others.
\end{abstract}

\section{Theories of Contexts}

In the AI literature, the term context has been applied to a profusion of ideas that have not been clearly distinguished. Some of them concern the syntactic representation of contexts; others refer to the semantic relationship of a linguistic context to a physical situation; and still others introduce pragmatic notions concerning the purpose or use of a context in various applications. Each of these major areas can be subdivided further. Syntactically, there are three distinct aspects of context:

1. A mechanism for grouping, associating, or packaging information that can be named and referenced as a single unit.

2. The contents of that package, which have been called anything from quoted formula to microtheory.

3. The permissible operations on the information in the package and the constraints on importing and exporting information into and out of a package.

All three of these notions represent syntactic mechanisms for representing and manipulating logical formulas without any consideration of their relationship to the real world, a possible world, or some model of the world. Much of the controversy about contexts results from the lack of a formal semantics that relates these operations to a Tarski-style model. Even an informal semantics that displays the intuitive meaning of contexts in terms of real-world objects and situations would be helpful as a guide to further analysis and formalization.
Some of the confusion about contexts results from an ambiguity in the English word. Dictionaries list two major senses of the word context:

- The basic meaning is a section of the linguistic text or discourse that surrounds some word or phrase of interest.

- The derived meaning is a nonlinguistic situation, environment, domain, setting, background, or milieu that includes some entity, subject, or topic of interest.

These two informal senses suggest intuitive criteria for distinguishing the various functions of contexts:

- Syntax. The syntactic function of context is to group, delimit, or package "a section of linguistic text." Formally, a context behaves like the QUOTE operator in Lisp together with the parentheses that delimit the portion of text that is quoted.

- Semantics. The quoted text of a context refers to something, which may be a physical entity or situation, a mathematical construction, or some other expression in a natural or artificial language.

- Pragmatics. The word interest, which occurs in both senses of the English definition, suggests some reason or purpose for distinguishing "a section of linguistic text" or "a nonlinguistic situation." That purpose constitutes the pragmatics or the reason why the text is being quoted. In Lisp, the QUOTE operator blocks the execution of the standard Lisp interpreter to allow nonstandard oper- 
ations to be performed for some other purpose. In logic, a quote blocks the standard rules of inference and allows the definition of new rules for some special purpose.

As this analysis indicates, the notion of context is intimately connected with a complex of related ideas. Much of the confusion results from which of them happens to be called a context: some people apply the word to the package; and others to the information contained in the package, to the thing that the information is about, or to the possible uses of either the information or the thing. The ideas themselves may be compatible, but they must be carefully distinguished and sorted out.

These intuitive criteria provide a basis for analyzing John McCarthy's (1993) "Notes on Formalizing Context" and relating the ideas to the other theories. McCarthy's basic notation is the predicate ist $(c, p)$, which may be read "the proposition $p$ is true in context $c$. " In his dissertation written under McCarthy's direction, R. V. Guha (1991) applied McCarthy's approach to the problem of partitioning a large, monolithic knowledge base into a collection of smaller, more modular microtheories. Guha implemented the microtheories in the Cyc system (Lenat \& Guha 1990), in which they have become a fundamental mechanism for organizing and structuring a knowledge base. McCarthy and Buvač (1994) have also applied contexts and the ist predicate to the analysis and representation of natural language discourse.

Although McCarthy, Guha, and Buvač have shown that the ist predicate can be a powerful tool for building knowledge bases and analyzing discourse, they have not clearly distinguished the syntax of contexts and propositions from their semantic relationship to some domain of discourse. In fact, the ist predicate itself mixes the syntactic notion of containment (is-in) with the semantic notion of truth (is-true-of). To clarify these relationships, it may be helpful to analyze the ist predicate as a conjunction of three more primitive predicates, is-in, refers-to, and describes:

$$
\begin{aligned}
& \text { ist }(c, p) \equiv(\exists x: \text { Entity })(\text { is-in }(c, p) \wedge \\
& \text { refers-to }(c, x) \wedge \text { describes }(x, p)) .
\end{aligned}
$$

According to this analysis, the proposition $p$ is true in context $c$ if and only if there exists some entity $x$ such that $p$ is in $c, c$ refers to $x$, and $p$ describes $x$. The formula distinguishes the abstract context $c$ from some nonlinguistic entity $x$, which represents the "situation, environment, domain, setting, background, or milieu" associ- ated with $c$. The predicate is-in represents the syntactic relationship of $c$ to $p$; and the predicates refers-to and describes represent the semantic relationships of $c$ and $p$ to the external entity $x$. McCarthy, Guha, and Buvac have primarily considered the syntactic operations associated with the is-in component of the ist predicate. To justify those operations, the semantics of the refers-to and describes components must also be addressed.

Much of the controversy about contexts results from the abundance of notation and terminology in different theories, their application to diverse phenomena, and the lack of communication between the different schools of thought. The purpose of this paper is to emphasize the underlying similarities and to promote crossfertilization of ideas. The following five theories will be considered:

1. Charles Sanders Peirce (1885) invented the modern algebraic notation for predicate calculus; but a dozen years later, he developed an alternate notation, which he called existential graphs (Roberts 1973). Although Peirce's algebra and graphs had equivalent expressive power, the graphic structure served as a heuristic aid that led him to explore operations and applications that were overlooked by logicians who used only the algebraic notation. In particular, Peirce's graphic notation for contexts was isomorphic to the discourse representation structures (DRSs) invented by Hans Kamp eighty years later. His rules of inference were based on operations of iterating and deiterating information to and from contexts in a way that resembles John McCarthy's lifting rules.

2. Hans Kamp (1981) developed discourse representation theory (DRT) to express the logical constraints on anaphoric references in natural language. Because of the difficulty of expressing those constraints in the algebraic notation for logic, Kamp introduced the graphic DRS notation, which allowed a simpler formulation of his rules. Significantly, the nested contexts in Kamp's DRSs are isomorphic to the nest of contexts in Peirce's EGs, even though Kamp had no previous knowledge of them. Kamp deserves credit for discovering the constraints on anaphora in DRT, but DRSs and EGs are equally suitable for expressing those constraints.

3. Jon Barwise and John Perry (1983) developed situation semantics as a theory of meaning in natural language. Unlike 
Montague's approach (1975), which related the semantics of language to potentially infinite models of the real world or possible worlds, Barwise and Perry adopted finite situations as their basis. Each situation is a bounded region of space-time containing physical objects and processes, as well as other situations. A great deal of research has been done within the paradigm of situation semantics (Barwise et al. 1991), including efforts to merge it or at least reconcile it with DRT (Cooper \& Kamp 1991). An important question is how and whether it can be merged or reconciled with McCarthy's contexts as well.

4. John Sowa (1984) developed conceptual graphs as a system of logic and reasoning based on the semantic networks of $\mathrm{AI}$ and the existential graphs of C. S. Peirce. The nodes called concepts correspond to typed, quantified variables in a sorted predicate calculus. A context is a defined as a concept of type Proposition, whose referent field contains one or more conceptual graphs that state the proposition. Later papers (Sowa \& Way 1986; Sowa 1991) used the CG contexts to represent Kamp's DRSs and Barwise and Perry's situations. In a paper on "Crystallizing Theories out of Knowledge Soup," Sowa (1990) proposed the use of contexts for partitioning a knowledge base into a collection of smaller "chunks" that could be assembled into theories appropriate to any particular application. In his dissertation, Guha (1991) cited the knowledge soup paper, which he said was "in the same spirit as the work described in this document."

\section{Peirce's Contexts}

First-order predicate calculus was independently invented by Gottlob Frege (1879) and Charles Sanders Peirce (1885). Frege used a tree notation, which no one else ever adopted. But Peirce developed an algebraic notation, which through the textbook by Emst Schröder (1890) and with a change of symbols by Giuseppe Peano became the modern system of predicate calculus. Long before Bertrand Russell learned logic from Frege and Peano, it had become a flourishing subject based on the Peirce-Schröder foundations.

The early history of modern logic is a fascinating tale that has been recounted by Roberts (1973) and Houser et al. (1995). The main point for this paper is that the man who invented the
5. John McCarthy is one of the founding fathers of AI, whose collected work (McCarthy 1990) has frequently inspired and sometimes revolutionized the application of logic to knowledge representation. His work on context, although published later than the previous four approaches, has grown out of ideas based on his earlier work. McCarthy's ist predicate is the key to relating that work to the ongoing research in the other paradigms. If the ist predicate can be defined in terms of the other theories, then any results obtained in one approach can be translated to any of the others. Besides defining contexts, McCarthy has been emphasizing his lifting rules for importing and exporting information into and out of the quoted text or package. Such rules, which resemble Peirce's rules of iteration and deiteration, are essential for allowing quoted information to be unquoted and used.

Besides these five theories, there is a long history of related ideas in logic, philosophy, linguistics, and AI. The most important ones for a theory of context include indexicals, possible worlds, metalanguage, belief revision, and ontology revision. Yet the various ideas and theories were developed by people with different intuitions, which they applied to different problems of knowledge representation. Trying to unify and clarify those intuitions by defining the terms of one theory in those of another runs the risk of distorting the insights of both. This paper will explore the implications of these definitions to determine whether the benefits of unification and clarification outweigh any possible distortions of the original insights.

common algebraic notation for logic later abandoned it for a graph representation, which he called his "chef d'oeuvre" and "the luckiest find of my career." With the existential graphs that he invented in 1897, Peirce developed an aspect of logic that was largely ignored by the mathematical logicians of the twentieth century. In relating Peirce's later logic and philosophy to situation semantics, Burke (1991) said "Peirce anticipated in his own way some of the concerns of situation theory (or rather, he happened to be working before it went out of fashion to wrestle with such concerns)." A century later, those concerns are back in fashion, and Peirce is once again in the avant garde of modern logic. 
The three primitives of existential graphs (EGs) include the oval enclosure, which delimits a context, the line of identity, which corresponds to an existentially quantified variable, and juxtaposition, which represents conjunction. The default interpretation of an oval with no other qualifiers is negation of the graphs nested inside. Existence, conjunction, and negation provide a complete representation for all of first-order logic. As an example, the middle diagram in Figure 1 is an existential graph for the sentence If a farmer owns a donkey, then he beats it.

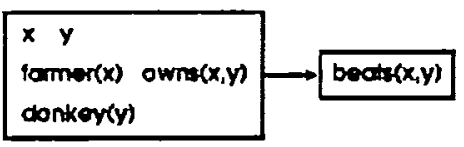

Discourse Representation Structure

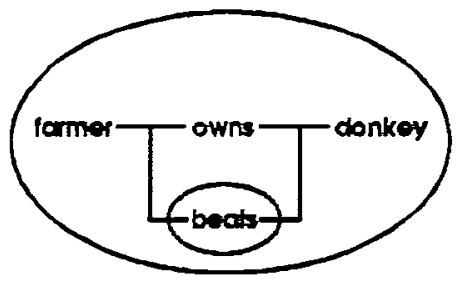

Exbtential Groph

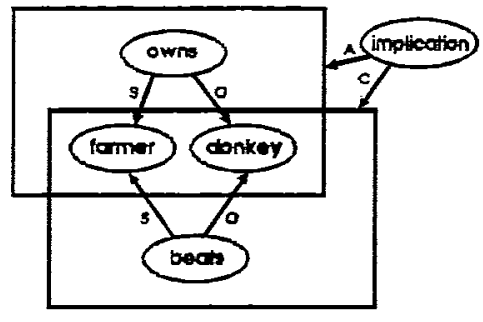

Pattlioned Semantle Network

Figure 1. Three representations for "If a farmer owns a donkey, then he beats it."

The EG in Figure 1 has two ovals, which represent negations. It also has two lines of identity, represented as linked bars: one line, which connects farmer to the left side of owns and beats, represents an existentially quantified variable $(\exists x)$; the other line, which connects donkey to the right side of owns and beats represents another variable $(\exists y)$. When Figure 1 is translated to the algebraic notation, farmer and donkey map to monadic predicates; owns and beats map to dyadic predicates. The implicit conjunctions can be represented with Peano's symbol $\wedge$ :

$$
\begin{aligned}
& \sim(\exists x)(\exists y)(\operatorname{farmer}(x) \wedge \text { donkey }(y) \wedge \\
& \text { owns }(x, y) \wedge \sim \text { beats }(x, y)) .
\end{aligned}
$$

Peirce called a nest of two ovals, as in Figure 1, a scroll, which he used to represent material implication, since $\sim(p \wedge \sim q)$ is equivalent to $p \supset q$. Using the $\supset$ symbol, the above formula may be rewritten as

$$
\begin{aligned}
& (\forall x)(\forall y)((f a r m e r(x) \wedge \text { donkey }(y) \wedge \\
& \text { owns }(x, y)) \supset \text { beats }(x, y)) .
\end{aligned}
$$

The algebraic formula with the $\supset$ symbol illustrates a peculiar feature of logic in comparison with natural languages: in order to preserve scope, the implicit existential quantifiers in the phrases a farmer and a donkey must be moved to the front of the formula and be translated to universal quantifiers. This puzzling feature of logic has posed a problem for linguists. In his discourse representation structures, Hans Kamp (1981) resolved it by introducing a new symbol for implication with different scoping rules. The diagram on the left of Figure 1 shows a DRS for the donkey sentence. The two boxes connected by an arrow represent the English pair if-then.
The variables $x$ and $y$ in the antecedent box have implicit existential quantifiers; Kamp defined the scoping rules for the DRS to include consequent box within the scope of the antecedent. As in existential graphs, conjunction is implicitly shown by juxtaposition. Altogether, the DRS may be read If there exists a farmer $x$ and $a$ donkey $y$ and $x$ owns $y$, then $x$ beats $y$.

Although the DRS and EG notations look quite different, they are exactly isomorphic: they have the same three primitives and exactly the same scoping rules for variables or lines of identity. What makes this coincidence remarkable is that in the dozens of notations for semantic networks in the 1960s and 1970s, no one else rediscovered Peirce's conventions. The notation that comes closest is the partitioned semantic network by Gary Hendrix (1975), which is illustrated in the rightmost diagram of Figure 1. Like Peirce and Kamp, Hendrix took the existential quantifier as the default, represented conjunction by juxtaposition, and used a graphic enclosure for partitioning contexts. But unlike Peirce and Kamp, Hendrix allowed overlapping contexts: the two overlapping boxes in Figure 1 represent the antecedent and the consequent of the implication.

With overlapping contexts, Hendrix had no need for scoping rules. Although the farmer and donkey nodes each occurred only once, the overlap allowed them to occur simultaneously in both contexts. Yet the overlapping contexts proved to be unwieldy: with more than three contexts, it became impossible to draw partitioned nets on a plane. Furthermore, the nesting of clauses in 
natural languages has a more direct mapping to the Peirce-Kamp nested contexts than to Hendrix's overlapping contexts. Kamp's rules for resolving anaphora in DRSs could be stated equally well in terms of EGs, but not in terms of overlapping contexts.

Besides notation, Peirce defined rules of inference for EGs, which in many respects are the simplest and most elegant inference rules ever devised for any version of logic. A typical theorem that requires 43 steps to prove with Russell and Whitehead's rules of 1910 takes only 8 steps with Peirce's rules of 1897 . Peirce's rules are a generalization of natural deduction, which Gerhard Gentzen discovered 40 years later. Like Gentzen, Peirce took the empty set as his only axiom, but Peirce's proofs are simpler than Gentzen's because the nesting of contexts eliminates the bookkeeping needed for making and discharging assumptions - the most error-prone aspect of Gentzen's system. For further dis- cussion of these points, see Roberts (1973), Sowa (1984, 1993), and Houser et al. (1995).

In discussing modality, Peirce imagined the graphs drawn on "a book of separate sheets, tacked together at points." The upper sheet represents "a universe of existing individuals," while the other sheets "represent altogether different universes with which our discourse has to do." Graphs on those sheets may represent "conceived propositions which are not realized." Peirce said that a necessarily true proposition could be considered as replicated on all the sheets in the book, while a possible proposition might occur on only one. In the algebraic notation, Peirce used the symbol $\omega$ as an index for "a state of things," to which he applied a universal quantifier for necessity and an existential quantifier for possibility. With his interpretation of necessity as truth "under all circumstances," Peirce was following Leibniz and anticipating Kripke.

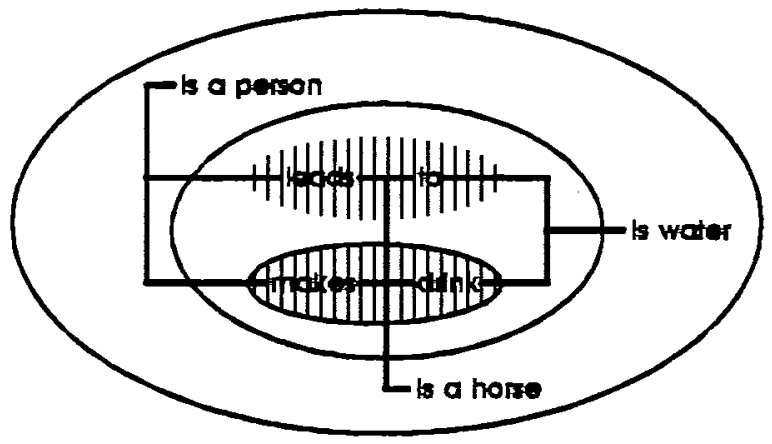

Figure 2. EG for "You can lead a horse to water, but you can't make him drink."

In 1906, Peirce introduced colors or tinctures to represent modalities. Figure 2 shows one of Peirce's examples, but with shading instead of the original red for possibility. The graph contains four ovals: the outer two are associated to form a scroll for if-then; the inner two represent possibility (shading) and impossibility (shading inside a negation). The outer oval may be read If there exists a person, a horse, and water, the next oval may be read then it is possible for the person to lead the horse to the water and not possible for the person to make the horse drink the water.

The notation_leads_to_represents the triadic predicate leads-to $(x, y, z)$, and _makes_drink_represents makes-drink $(x, y, z)$. In the algebraic notation with $\diamond$ for possibility, Figure 2 maps to the following formula:

$$
\begin{aligned}
& \sim(\exists x)(\exists y)(\exists z)(\text { person }(x) \wedge \text { horse }(y) \wedge \\
& \text { water }(z) \wedge \sim(\diamond \text { leads-to }(x, y, z) \wedge \\
& \sim \diamond \text { makes-drink }(x, y, z))) .
\end{aligned}
$$

With the symbol $\supset$ for implication, this formula becomes

$$
\begin{aligned}
& (\forall x)(\forall y)(\forall z)((\operatorname{person}(x) \wedge \text { horse }(y) \wedge \\
& \text { water }(z)) \supset(\Delta \text { leads-to }(x, y, z) \wedge \\
& \sim \Delta \text { makes-drink }(x, y, z))) .
\end{aligned}
$$

This version may be read For all $x, y$, and $z$, if $x$ is a person, $y$ is a horse, and $z$ is water, then it is possible for $x$ to lead $y$ to $z$, and not possible for $x$ to make $y$ drink $z$.

As a systematic way of representing the kinds of contexts, Peirce adopted the traditional heraldic tinctures, which were classified as metal, color, or fur. He applied that three-way distinction to actual, modal, and intentional contexts: 
1. Metal: argent, or, fer, and plomb. Peirce used argent (white background) for "the actual or true in a general or ordinary sense," and the other metallic tinctures for "the actual or true in some special sense." A statement about the physical world, for example, would be actual in an ordinary sense. Peirce also considered mathematical idealizations, such as Cantor's hierarchy of infinite sets, to be "actual," but not in the same sense as ordinary physical entities.

2. Color: azure, gules, vert, and purpure. Peirce distinguished four basic modalities: azure for logical possibility (dark blue) and subjective possibility (light blue); gules for objective possibility; vert for "what is in the interrogative mood"; and purpure for "freedom or ability." Each of these modalities could be combined with negation: he defined necessary in the usual way as not-possibly-not and obligatory as not-freedom-not (an anticipation of deontic logic).

3. Fur: sable, ermine, vair, and potent. The four furs correspond to propositional attitudes: sable for "the metaphysically, or rationally, or secondarily necessitated"; ermine for purpose or intention; vair for "the commanded"; and potent for "the compelled."

Peirce's three-way classification is highly suggestive, but incomplete. He wrote that the complete classification of "all the conceptions of logic" was "a labor for generations of analysts, not for one." But throughout his analyses, he clearly distinguished the logical operators represented by the graphs, from the tinctures, which, he said, do not represent

differences of the predicates, or significations of the graphs, but of the predetermined objects to which the graphs are intended to refer. Consequently, the Iconic idea of the System requires that they should be represented, not by differentiations of the Graphs themselves but by appropriate visible characters of the surfaces upon which the Graphs are marked.

It seems that Peirce did not consider the tinctures to be part of logic itself, but of the metalanguage for describing how the logic applies to the universe of discourse:

The nature of the universe or universes of discourse (for several may be referred to in a single assertion) in the rather un- usual cases in which such precision is required, is denoted either by using modifications of the heraldic tinctures, marked in something like the usual manner in pale ink upon the surface, or by scribing the graphs in colored inks.

By 1906, mathematical logic based on Peirce's algebraic notation had become a flourishing field of research, and his graphs were ignored. There were several reasons for the neglect: the notation and terminology were unfamiliar; most logicians, who had a strong background in mathematics, had already found the algebraic notation congenial to their tastes; and significantly, Peirce's novel applications of his graph logic to modality, intentionality, and metalanguage were outside the main interests of the logicians of his time. Today, however, Peirce's contributions are central to research on contexts:

1. Representation of contexts by nests of enclosures, which separate or partition groups of propositions of different modal status.

2. First-order logic based on three operators: existence (line of identity), conjunction (juxtaposition), and negation (oval enclosure on a white background).

3. Sound and complete rules of inference for first-order logic based on operations of drawing or erasing graphs and importing or exporting graphs into and out of contexts.

4. Tinctures for distinguishing the purpose or "nature" of a context from its logical operators (for which he used only the basic three - existence, conjunction, and negation).

5. A three-way classification of the use of contexts for representing actuality (metal), modality (color), or intentionality (fur).

6. The use of graphs as a metalanguage for talking about graphs.

7. Complete statement of the rules of inference for existential graphs in existential graphs themselves.

Peirce's later writings, although fragmentary, incomplete, and mostly unpublished, are no more fragmentary and incomplete than many modern publications about contexts. Although (or perhaps because) he did not use the word context, Peirce was more consistent in distinguishing the syntax (oval enclosures), the semantics ("the universe or universes of discourse"), and the pragmatics (the tinctures that "denote" the "nature" of those universes). 


\section{Contexts in Conceptual Graphs}

Conceptual graphs are extensions of existential graphs with new features based on the semantic networks of $\mathrm{AI}$ and the linguistic research on thematic roles and generalized quantifiers. The primary difference is in the treatment of lines of identity. In existential graphs, the lines serve two different purposes: they represent existential quantifiers, and they show how the arguments are connected to the relations. In conceptual graphs, those two functions are split: boxes called concepts contain the quantifiers, and arcs marked with arrows show the connections of arguments to circles called conceptual relations. This separation of functions has several important consequences:

- Concepts have a place to represent a type label for each quantifier. Conceptual graphs therefore correspond to a typed or sorted logic, unlike the untyped existential graphs.

- Concepts may also contain a name or other specification of the referent of the concept, as in [Cat: Yojo], where the type is Cat and the particular individual is named Yojo. The area on the left of the colon is called the type field, and the area on the right is called the referent field.

- When an existential graph or an untyped formula in predicate calculus is mapped to a conceptual graph, the type label $T$ may be used to mark the universal type, which is a supertype of all others. The type $T$ imposes no restrictions on the quantifier or referent. The concept [ $T$ : Yojo], for example, would represent an entity named Yojo whose type was unknown or unspecified.

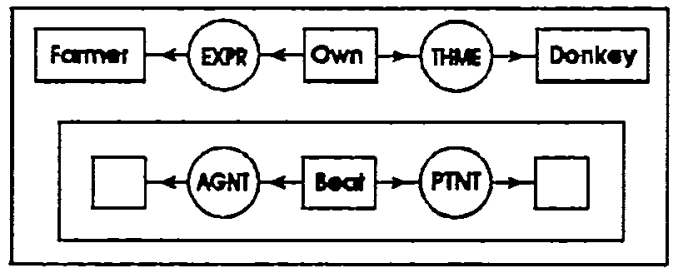

- The arrows or numbers on the arcs of conceptual relations distinguish the arguments more clearly than Peirce's unlabeled lines. For dyadic relations, the arrow pointing towards the circle is the first argument, and the arrow pointing away is the second argument. For relations with more than two arguments, the arcs are numbered $1,2, \ldots, n$; the arrow on the $n$-th arc points away from the circle, and the other arcs point towards the circle.

- In an existential graph, any point on a line of identity could be considered as a separate quantified variable. Conceptual graphs concentrate the point of quantification in the concepts rather than the lines. A blank referent field represents the quantifier $\exists$, but the universal quantifier $\forall$ or other generalized quantifiers may also occur in the referent field of a concept.

- Peirce's lines of identity could cross context boundaries, but a concept may only occur in a single context. When two concepts in different contexts refer to the same individual, they must be associated by coreference labels or by a dotted line called a coreference link.

To illustrate these features, Figure 3 shows two equivalent conceptual graphs for the donkey sentence. The CG on the left uses the basic notation with the $\neg$ symbol to mark negation and with dotted lines for coreference links. The CG on the right uses an extended notation with the types If and Then defined as negated propositions and with coreference shown by the labels $x$ and $y$. The concepts marked * $\mathrm{x}$ and ${ }^{*} \mathrm{y}$ are the defining nodes with implicit existential quantifiers, and the concepts marked ?x and ?y are bound nodes within the scope of $* x$ and $* y$.

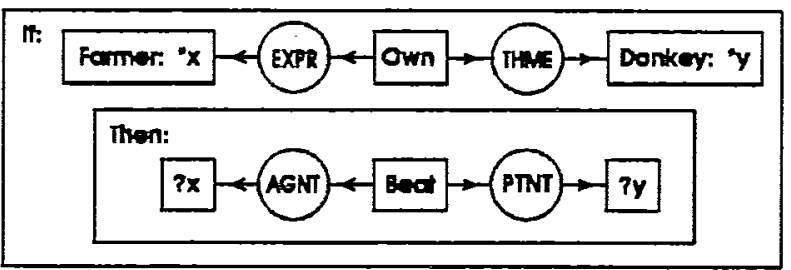

Figure 3. Two conceptual graphs for "If a farmer owns a donkey, then he beats it."

The scoping rules for the CGs in Figure 3 are the same as the rules for the DRS and EG in
Figure 1. The CGs may be read If a farmer $x$ owns a donkey $y$, then $x$ beats $y$. The circles rep- 
resent dyadic conceptual relations, where the arrow pointing towards the circle marks the first argument and the arrow pointing away marks the second argument. The two CGs in Figure 3 would correspond to the following formula in algebraic notation:

$$
\begin{aligned}
& \sim(\exists x: \text { Farmer })(\exists y: \text { Donkey })(\exists z: \text { Own })(\operatorname{expr}(z, x) \\
& \wedge \operatorname{thme}(z, y) \wedge \sim(\exists w: \text { Beat })(\operatorname{agnt}(w, x) \\
& \wedge \operatorname{ptnt}(w, y))) .
\end{aligned}
$$

This representation follows C. S. Peirce and Donald Davidson in reifying verbs with the event variables $z$ and $w$. The dyadic relations represent the thematic roles or case relations used in linguistics: experiencer (EXPR); theme (THME); agent (AGNT); and patient (PTNT). For convenience, there is also a linear notation that makes CGs easier to type:

$$
\begin{aligned}
& \neg[\text { [Farmer: *x }] \leftarrow(\text { EXPR }) \leftarrow[\text { Own }] \rightarrow(\text { THME }) \rightarrow[\text { Donkey: *y }] \\
& \neg[[? \mathrm{x}] \leftarrow(\text { AGNT }) \leftarrow[\text { Beat }] \rightarrow(\text { PTNT }) \rightarrow[\text { ?y }]]], \\
& {[\text { If : }[\text { Farmer: *x }] \leftarrow(\text { EXPR }) \leftarrow[\text { Owm }] \rightarrow(\text { THME }) \rightarrow[\text { Donkey: *y }]} \\
& [\text { Then: }[? \mathrm{x}] \leftarrow(\text { AGNT }) \leftarrow[\text { Beat }] \rightarrow(\text { PTNT }) \rightarrow[\text { ?y }]]] .
\end{aligned}
$$

In the graphic form of Figure 3, coreference may be shown by dotted lines, but coreference labels must be used in the linear notation.

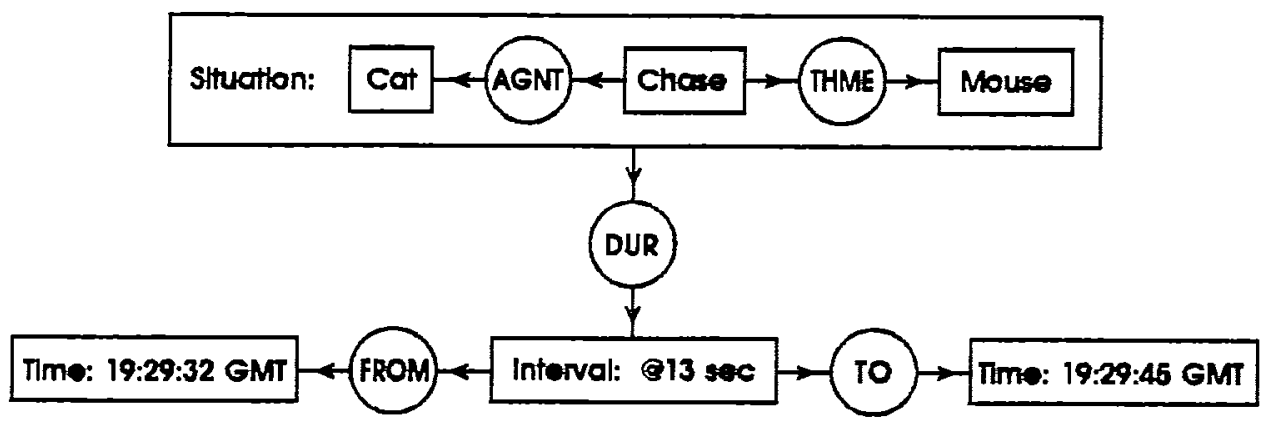

Figure 4. CG for "A cat chased a mouse for an interval of 13 seconds from 19:29:32 GMT to 19:29:45 GMT."

In CGs, a context is defined as a concept whose referent field contains nested conceptual graphs. Since every context is also a concept, it can have a type label, coreference links, and attached conceptual relations. In Figure 4, the graph for a cat chasing a mouse is nested inside a concept of type Situation. The conceptual graph in the inner context describes the situation. Attached to that context is the relation DUR for duration, which is linked to a concept for an interval of 13 seconds. The relations FROM and TO show that the interval lasted from 19:29:32 GMT to 19:29:45 GMT.

When a conceptual graph occurs in a context of type Graph, it is used as a literal; in a context of type Proposition, it states a proposition; in a context of type Situation, it describes a situation. The following concept, by itself, may be read $A$ situation of a cat chasing a mouse.

[Situation: [Cat] $+($ AGNT $)+[$ Chase $] \rightarrow($ THME $) \rightarrow[$ Mouse $]]$.

This graph may be considered an abbreviation for the following graph, which says that there exists a situation whose description (DSCR) is the proposition that a cat is chasing a mouse:

[Situation] $\rightarrow($ OSCR $) \rightarrow$ [Proposition:

$[$ Cat $] \leftarrow($ AGNT $) \leftarrow[$ Chase $] \rightarrow($ THME $) \rightarrow[$ Mouse $]]$.
In most applications, the abbreviated form shown in Figure 4 is used. When necessary, the type label of the context determines how the nested graph is interpreted. This use of the type label corresponds to type coercion in programming languages.

To relate Figure 4 to McCarthy's ist predicate, first expand the abbreviations; then translate the expanded graph to predicate calculus:

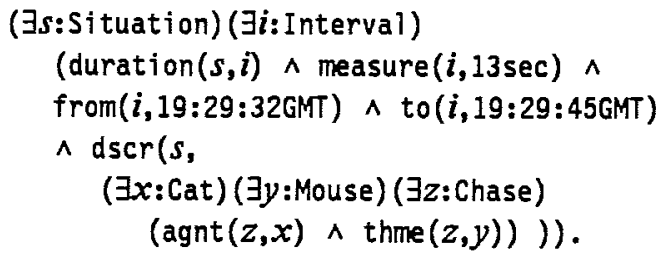

Now it is possible to relate parts of this formula to the earlier definition of the ist predicate:

$$
\begin{aligned}
& \text { ist }(c, p) \equiv(\exists x: \text { Entity })(\text { is-in }(c, p) \wedge \\
& \quad \text { refers-to }(c, x) \wedge \text { describes }(x, p)) .
\end{aligned}
$$

The entity $x$ can be identified with the situation $s$, and the proposition $p$ with the proposition that a cat is chasing a mouse. But there is no entity in this translation that can be identified with McCarthy's context $c$. In McCarthy's sense, $c$ is supposed to be larger than the single proposition. In fact, the context may be so rich that no finite set of formulas could exhaust its full content. 
This discussion raises a question about the distinction between a total description of everything that is knowable about a situation and a partial description in a single formula. That issue has been central to situation semantics, which may help to clarify the semantics of both CG contexts and McCarthy's contexts.

\section{Situations and Propositions}

Barwise and Perry (1983) developed situation semantics as a reaction against the potentially infinite models of Kripke's and Montague's modal and intensional logics. Each situation is a finite configuration of some aspect of the world in a limited region of space and time. It may be a static configuration that remains unchanged for a period of time, or it may include processes and events that are causing changes. It may include people and things with their actions and thoughts; it may be real or imaginary; and its time may be present, past, or future. A situation may be as large as the solar system or as small as an atom, and it may contain nested situations for smaller or more detailed aspects of the world.

As an illustration of the way situations are related to partial descriptions, Figure 5 shows a concept of type Situation, which is linked to two images and a description. The image relation (IMAG) links the situation to two different kinds of images of that situation: a picture and the associated sound. The description relation (DSCR) links it to a proposition that describes some aspect of the situation, which is linked by the statement relation (STMT) to three different statements of the proposition in three different languages: an English sentence, a conceptual graph, and a formula in the Knowledge Representation Language (KIF) by Genesereth and Fikes (1992).

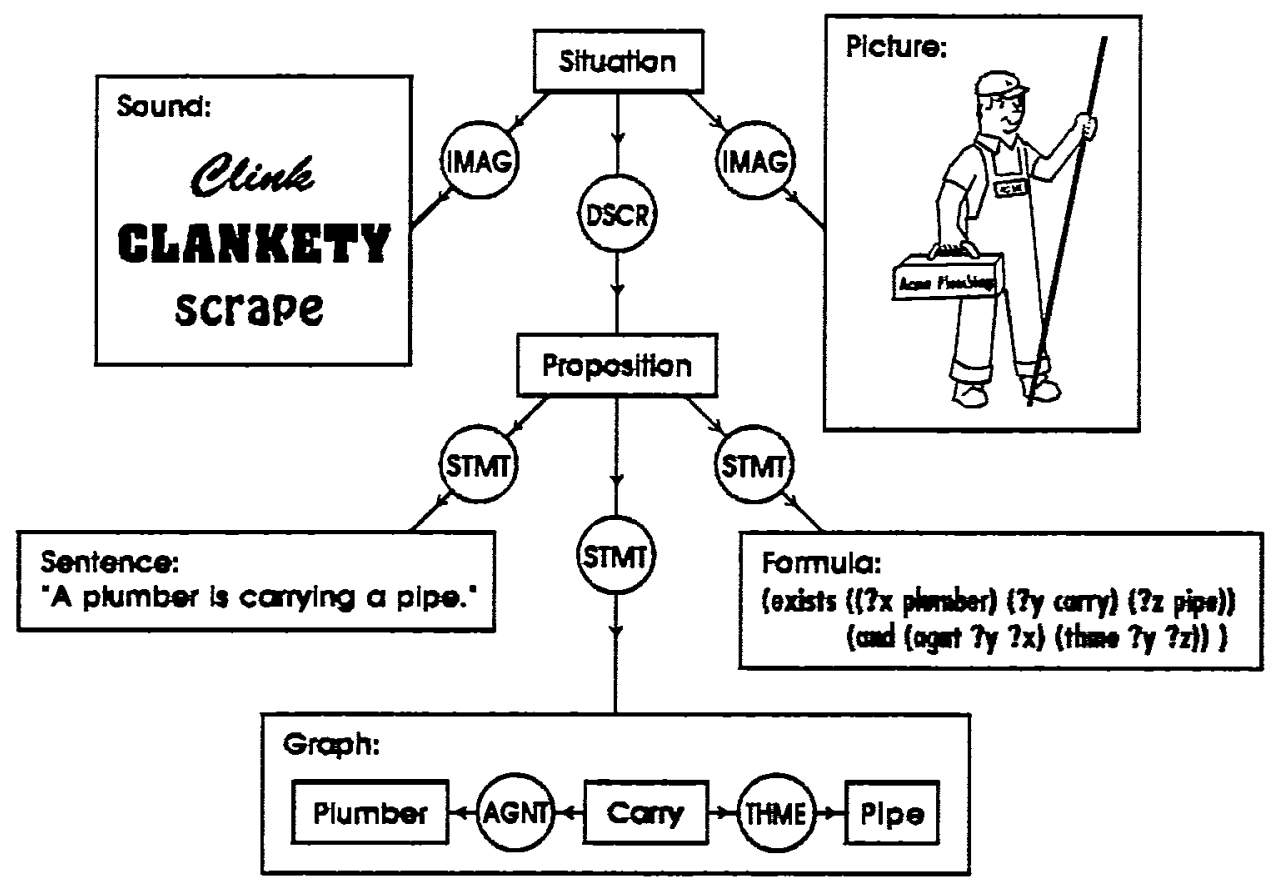

Figure 5. A situation of a plumber carrying a pipe

A proposition may be defined as an equivalence class of statements in one or more languages. No concrete statement in any specific language is the proposition. Instead, a concept of type Proposition, such as the one in Figure 5, represents a class of intertranslatable statements in one or more concrete languages. Each statement in that class is said to express the same proposition. To avoid complexities, the equivalence mapping will be defined first for formal languages like KIF and CGs. Let $f$ be a mapping from language $\mathscr{R}_{1}$ to language $\mathscr{L}_{2}$ that defines the equivalence; let $s$ be any statement in $\mathscr{L}_{1}$; and let $t=f^{-1}(f(s))$ be the result of mapping $s$ to $\mathscr{P}_{2}$ and back again. Then $f$ must obey the following three constraints: 
1. Truth preserving: The statements $s$ and $t$ must be provably equivalent according to the rules of inference of language $\mathscr{Z}_{1}$.

2. Vocabulary preserving: Exactly the same nonlogical symbols must appear in both $s$ and $t$.

3. Negation preserving: When $s$ and $t$ are mapped to Peirce Normal Form (with negation, conjunction, and the existential quantifier as the only logical operators), they contain exactly the same number of negation operators.

These three conditions ensure that the statements $s$ and $t$ are highly similar, if not identical. Although Fermat's Last Theorem and $2+2=4$ are both true, the proof is far from trivial, and the two statements require different vocabularies. Condition \#2 allows variables to be renamed and permits $p \vee q$ to be replaced by $\sim(\sim p \wedge \sim q)$; but it ensures that $a$ statement like $(\forall x)(\operatorname{dog}(x) \supset \operatorname{dog}(x))$ does not get mapped to $(\forall x)(\operatorname{cat}(x) \supset \operatorname{cat}(x))$. Condition \#3 allows $p \wedge p$ to be mapped to $p$, but it prevents $\sim \sim p$ from being mapped to $p$. Generalizing this definition to natural languages should be possible, but that would raise further issues beyond the scope of this paper.

As Figure 5 illustrates, the proposition stated by any of the three statements represents a tiny fraction of the total information available. Both the sound image and the picture image capture information that is not in the sentence, but even they are only partial representations. A picture may be worth a thousand words, but a situation can be worth a thousand pictures. To relate a situation to the information it contains, Keith Devlin (1991) introduced the term infon for an abstract information object that is closely related to the previous definition of proposition.

Devlin's basic formula is $s \models \sigma$, where $s$ is a situation and $\sigma$ is an infon that is semantically entailed by $s$. Devlin's construction that comes

\section{Extensions, Intensions, and Intentions}

The difficulty of giving a precise definition of McCarthy's contexts is closely related to the difficulty of giving a precise definition of situations. In their 1983 book, Barwise and Perry identified a situation with a bounded region of space-time, and they tried to use that definition to support a theory of propositional attitude verbs, such as believe, think, hope, fear, wish, want, and plan. Yet further research on situation semantics has closest to what McCarthy calls a context is the set of all infons entailed by a situation: $\{\sigma \mid$ $s \models \sigma\}$. If the DSCR relation is interpreted as entailment of a partial description, McCarthy's context $c$ may be considered a complete description of a situation:

$$
\text { completeDscr }(s, c) \equiv c=\{\sigma \mid s \models \sigma\} .
$$

For Figure 5 , the context $c$ would include propositions or infons saying that the plumber is carrying a toolbox, he works for Acme Plumbing Co., he is dragging the pipe with a clankety noise, etc. The predicate is-in $(c, p)$ could then be interpreted as provability.

With this analysis, the following formula represents an interpretation of the ist predicate in terms of situations and infons (or propositions):

$$
\begin{aligned}
& \text { ist }(c, p) \equiv(\exists s: \text { Situation) (provable }(c, p) \wedge \\
& \quad \text { completeDscr }(s, c) \wedge d \operatorname{dscr}(s, p)) .
\end{aligned}
$$

The syntactic predicate is-in $(c, p)$ is interpreted as provable $(c, p)$. The two semantic predicates are defined in terms of entailment: refers-to $(c, s)$ is completeDscr $(s, c)$; and $\operatorname{dscr}(s, p)$ is $s=p$. In a pure first-order framework, $s \neq p$ would be redundant, since it would be implied by $c \vdash p$ and the definition of $c$. Including it, however, could accommodate nonstandard logics in which provability is not equivalent to entailment.

The previous definition was presented at the UCAI'95 workshop on context. In the discussions, McCarthy made the point that identifying a context $c$ with the set of all entailments of a situation $s$ does not completely capture his intuitions about context. As an example, suppose that John believes everything that Mary believes. Then either of their belief systems would entail exactly the same propositions. But McCarthy would like to say that the context of John's beliefs is not the same as the context of Mary's beliefs. That distinction implies that no purely extensional definition can capture the full import of McCarthy's notion of context.

shown that a purely extensional definition of situation is inadequate, for many of the same reasons that McCarthy objected to an extensional definition of context. The following examples illustrate the problems:

- A college lecture might be considered a situation bounded by a 50-minute time period in a spatial region enclosed by the walls of a classroom. But if the time were moved for- 
ward by 30 minutes, the region would include the last half of one lecture and the first half of another. That time shift would create a very unnatural "situation."

- An even more unnatural transformation would be to shift the spatial region to the left by half the width of a classroom. Then it would include part of one class listening to one teacher, part of another class listening to a different teacher speaking on a different topic, and a wall that separated the two discussions.

- Another possible transformation is to fix the coordinate system relative to the sun instead of the earth. Then the space-time region that included the class at the beginning of the lecture would quickly shift into deep space as the earth moved; by the end of the hour, it would contain nothing but an occasional hydrogen atom.

These examples illustrate a fundamental difficulty for any purely extensional definition. An arbitrarily chosen space-time region is useless for defining a "situation" or "context." Instead, a useful or "meaningful" choice of region depends critically on some agent's purpose or intention. But if purpose or intention is critical to the definition of situation or context, then the explication of propositional attitudes in terms of situations becomes circular: the intentionality that is explained by the theory depends on the intentionality that went into the choice of situation.

Peirce considered intentionality as fundamental to his theory of signs or semiotics. Although he did not use the word context, he would have defined a context as a kind of sign, to which he would apply his basic definition:

A sign, or representamen, is something which stands to somebody for something in some respect or capacity. It addresses somebody, that is, creates in the mind of that person an equivalent sign, or perhaps a more developed sign. That sign which it creates I call the interpretant of the first sign. The sign stands for something, its object. It stands for that object, not in all respects, but in reference to a sort of idea, which I have sometimes called the ground of the representamen. (CP 2.228)
Peirce's notion of sign was broad enough to include situations, contexts, propositions or infons, and their expression in any language, including English and logic. His notion of ground is crucial: it acknowledges that some agent's purpose, intention, or "conception" is essential for determining the scope of a situation or context.

Unlike the two-way distinction of extensions and intensions, Peirce drew a three-way distinction in his basic categories of Firstness, Secondness, and Thirdness:

First is the conception of being or existing independent of anything else. Second is the conception of being relative to, the conception of reaction with, something else. Third is the conception of mediation, whereby a first and a second are brought into relation.

His three kinds of tinctured contexts, which were discussed in Section 2, are applications of these categories:

1. Metal represents the "Actual" or what can be described in classical first-order logic without modalities or intentionality.

2. Color represents the varieties of possibilities, objective, subjective, and deontic. This topic has been thoroughly explored in the modern modal and intensional logics, whose semantics have been defined in terms of infinite families of possible worlds.

3. Fur represents the intentionality (with a $T$ instead of an S) whereby an agent selects some context for the representation of some object for some purpose. Intentionality, in Peirce's terms, is the mediating Thirdness that determines the context for directing attention to some object for some purpose.

Peirce's third category has never been adequately studied or represented in any of the modern modal and intensional logics. It is central to the issues that McCarthy, Barwise and Perry, and others have been trying to capture in their theories. But those theories cannot be completed without representing intentionality with a $T$. Peirce never completed his theory of intentionality, but at least he made a good beginning. As he said, the complete classification of all the conceptions is "a labor for generations of analysts, not for one." 


\section{References}

Barwise, Jon, Jean Mark Gawron, Gordon Plotkin, \& Syun Tutiya, eds. (1991) Situation Theory and its Applications, CSLI, Stanford, CA.

Barwise, Jon, \& John Perry (1983) Situations and Attitudes, MIT Press, Cambridge, MA.

Burke, Tom (1991) "Peirce on truth and partiality," in Barwise et al. (1991) pp. 115-146.

Cooper, Robin, \& Hans Kamp (1991) "Negation in situation semantics and discourse representation theory," in Barwise et al. (1991) pp. 311-333.

Devlin, Keith (1991) "Situations as mathematical abstractions," in Barwise et al. (1991) pp. 25-39.

Frege, Gottlob (1879) Begriffsschrift, translated in Jean van Heijenoort, ed. (1967) From Frege to Gödel, Harvard University Press, Cambridge, MA, pp. 1-82.

Genesereth, Michael R., \& Richard E. Fikes (1992) Knowledge Interchange Format, Reference Manual, Version 3.0, Report Logic-92-1, Computer Science Department, Stanford University.

Guha, R. V. (1991) Contexts: A Formalization and Some Applications, technical report ACT-CYC-423-91, MCC, Austin, TX.

Houser, N., D. D. Roberts, \& J. Van Evra, eds. (1995) Studies in the Logic of Charles Sanders Peirce, Indiana University Press, Bloomington.

Kamp, Hans (1981) "Events, discourse representations, and temporal references," Langages 64, 39-64.

Lenat, Douglas B., \& R. V. Guha (1990) Building Large Knowledge-Based Systems, AddisonWesley, Reading, MA.

McCarthy, John (1990) Formalizing Common Sense, Ablex, Norwood, NJ.

McCarthy, John (1993) "Notes on formalizing context," Proc. IJCAI-93, Chambéry, France, pp. $555-560$.

McCarthy, John, \& Saša Buvač (1994) Formalizing Context, Technical Note
STAN-CS-TN-94-13, Stanford University. Available from http://sail.stanford.edu.

Peirce, Charles Sanders (1885) "On the algebra of logic," American Journal of Mathematics, vol. 7, pp. 180-202. Reprinted in Peirce (W) vol. 5.

Peirce, Charles Sanders (CP) Collected Papers of C. S. Peirce, ed. by C. Hartshorne, P. Weiss, \& A. Burks, 8 vols., Harvard University Press, Cambridge, MA, 1931-1958.

Peirce, Charles Sanders (W) Writings of Charles S. Peirce, vols. 1-5, Indiana University Press, Bloomington, 1982-1993.

Roberts, Don D. (1973) The Existential Graphs of Charles S. Peirce, Mouton, The Hague.

Schröder, Ernst (1890-1895) Vorlesungen über die Algebra der Logik, 3 vols., Teubner, Leipzig. Reprinted by Chelsea Publishing Co., Bronx, NY, 1966.

Sowa, John F. (1984) Conceptual Structures: Information Processing in Mind and Machine, Addison-Wesley, Reading, MA.

Sowa, John F. (1990) "Crystallizing theories out of knowledge soup," in Z. W. Ras \& M. Zemankova, eds., Intelligent Systems: State of the Art and Future Directions, Ellis Horwood, New York, p. 456-487.

Sowa, John F. (1991) "Towards the expressive power of natural language," in J. F. Sowa, ed. (1991) Principles of Semantic Networks: Explorations in the Representation of Knowledge, Morgan Kaufmann Publishers, San Mateo, CA, pp. 157-189.

Sowa, John F. (1993) "Logical foundations for representing object-oriented systems," Journal of Experimental and Theoretical $A I$, vol. 5, nos. 2\&3, pp. 237-261.

Sowa, John F., \& Eileen C. Way (1986) "Implementing a semantic interpreter for conceptual graphs," IBM Journal of Research and Development 30:1, pp. 57-69. 